\title{
An Event-Driven System Based on Internet Oriented to Equipment Fault and Maintenance
}

\author{
Duan $\mathrm{Bin}^{1, \text { a }}$ \\ ${ }^{1}$ Wuxi CAS Ubiquitous Information Technology R\&D Center CO., LTD, Wuxi, JiangSu \\ adb19820409@163.com
}

Keywords: Internet; Event-Driven; Equipment Fault

\begin{abstract}
An event-driven system oriented to equipment detection as well as fault operation and maintenance is built. With the ability to provide remote access, the system which supports Internet Protocol can be integrated with workshop's manufacturing execution system as a sub-system so that the equipment operation and maintenance data will be no longer the information island.
\end{abstract}

\section{Introduction}

In recent years, not only has informatization construction drawn growing attention, but the position of information management software has been elevated considerably in the wake of the continuous enterprise development. The major function of equipment fault operation and maintenance system is to prevent, diagnose and maintain the faults of the equipment- the key production resource-in production. It is evident that this system is of vital importance. In most enterprises[1], however, equipment fault operation and maintenance system is only used internally. Experts will be only invited when equipment problems fail to be addressed[2]. This on the one hand will result in the failure to play the role of information system and on the other will delay the production progress and raise maintenance cost.

In this paper, an event-driven equipment failure operation and maintenance system based on B/S architecture has been designed. Based on the reasonable permission assignment, equipment experts may localize the faults by logging in to the operation and maintenance system via browser telnet[3]. In addition, by configuring events, partial functions in the system can be automatically triggered, and then, the system will become more intelligent to upgrade user experience.

\section{System structure}

Based on B/S architecture, the system equips front end with WPF, server side with WCF, and database with SQLServer, and besides, partial functions are developed for the mobile terminal as well. Figure 1 is system's structure chart.

The system mainly consists of five modules: field detection, data diagnosis, work order management, system model.

\section{System model}

Its aim is to build a digital static model for equipment, detection position, system event, login user, and user authority as the fundamental data that support operation and maintenance system[4]. System model includes the sub-modules such as equipment model, detection position model, event management model, user management, role management, and authority management.

Equipment model is to build a model for the information of production equipment in factory, such as about equipment name and type. Currently, all corporate information management systems are designed with equipment management function. Given the applicability and integration performance of the system, this module offers a synchronous integration interface to facilitate the integration with information management system. 
Detection position model is to build a model for the detection positions of equipment. For different types of equipment, the detection positions are different, and besides, the maintenance cycle varies from position to position. Defining detection position can make the maintenance task more clear and the maintenance record more specific, as well as provide data for equipment operation analysis.

Event management model is to build a model for the events in relation to operation and maintenance. Event is an abstract description of a series of associated behaviors. Event management module is comprised of front-end event management and configuration page, and backend event detection and processing services. Aimed at defining events, event management and configuration page includes event-triggering condition and post-event processing methods (DLL or stored procedure). Event detection service is intended to detect the event-triggering conditions in real time. Once the events are detected to have occurred, the configured processing methods in event model will be put into use. The events in system are mainly composed of work order notification, work order completion, fault early-warning, and fault alarm. In addition, secondary development is implemented in the light of actual business with the purpose of adding events to the system. When the event-triggering conditions and processing methods are completely configured, event service will be able to process the events accurately, which will contribute largely to the expansibility and applicability of the system. Figure 2 is the event module process:

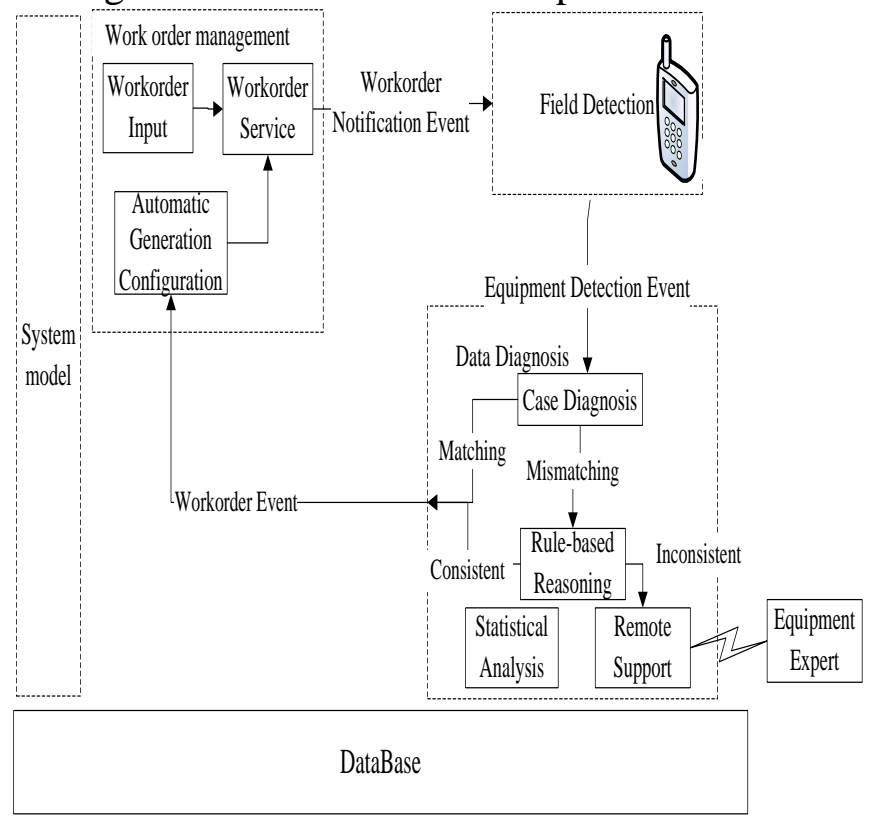

Fig.1. System's Structural Interaction Diagram

User management is aimed at managing system users and able to record user information such as account number, password, role, email and mobile phone number via interactive interface.

Role management is intended to categorize system users.

Authority management is aimed at assigning permission to a role; in this way, users will understand their rights and responsibilities clearly and then avoid system damage resulting from improper operations while using the system.

\section{Work order management}

Work order is the description about operation and maintenance task that involves the information such as operation time, target device, work order state, work order maker, work order executor, operation and maintenance type, detection position[5], and operating steps. Work order management consists of the work order input interface and the automatic generation configuration page in the front end, and the work order service in back-end server. 


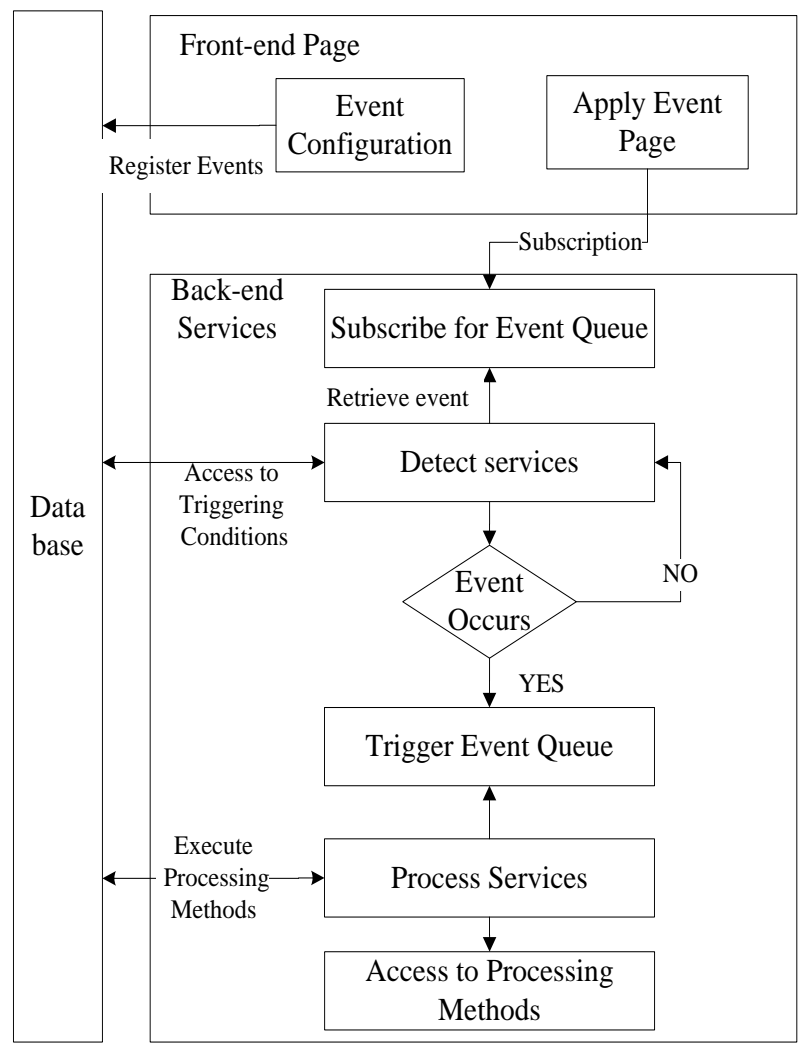

Fig. 2. Event Module Process

Authority management is aimed at assigning permission to a role; in this way, users will understand their rights and responsibilities clearly and then avoid system damage resulting from improper operations while using the system.

Work order input page generates an operation and maintenance task by inputting relevant information of work order via interactive interface;

Automatic generation configuration page is the module where work order is automatically generated. Though the module is equipped with work order input function, the formulation of work order will be surely a heavy task if there are large numbers of equipment and equipment types. Therefore, the module provides an automatic generation module in which the tasks input by hand are assigned to system, which will not only reduce work load, but also avoid the errors of manual input. The module is mainly comprised of generation cycle and the information required by work order;

Work order service function is to generate work order periodically according to the automatic generation module, and meanwhile send the work order to the responsible person of work order.

\section{Field detection}

Maintaining and detecting personnel detect and input the key parameters of equipment in the system according to the requirements of work order with the purpose of providing effective data for subsequent equipment analysis and diagnosis. Considering the complexity of on-site environment, convenience and applicability of equipment detection, and diversity of collection means, this module fully resorts to the portability and the multimedia functions of mobile terminals such as shooting and audio and video recording based on the development of mobile terminals. In this way, the detecting personnel will be able to collect the fault information from different dimensions, display the real faults, and localize the faults more effectively by means of multimedia functions. In addition, the system will record the information of the faults (such as fault phenomena, key parameter values of equipment and solutions) that have occurred in the historical library. Upon completion of fault collection, the system will match the information collected with those in historical library so as to assist the detecting personnel with the preliminary fault diagnosis. If there 
are matching records, the system will notify relevant responsible person of reconfirmation via message, email and system message (through system configuration), and besides[9], the system will remind relevant personnel of further analysis by sending a notification if there are no matching records.

An analysis on the field data collected is performed to figure out whether the equipment has a requirement for maintenance or repair as well as to provide relevant repair methods. The modules primarily consist of four sub-modules, including case diagnosis, rule-based reasoning, statistical analysis and remote support.

Case diagnosis analyzes the current situation of equipment by matching the data sample collected with those in case library. Case diagnosis involves matching engine and case library. Case library records the historical data of equipment maintenance and faults at browser side, and matching engine, which runs at server side, is intended to match samples and cases. Through event configuration, matching engine is capable of implementing automatic case diagnosis and work order generation; that is, case diagnosis will start automatically upon the completion of field detection, and if the matching is a success, corresponding work order will be generated automatically and sent to relevant responsible person for examination. Despite the advantage that has no need for manual interaction, case diagnosis, aimed at the deterministic matching of historical data, also shows some deficiency, i.e, lack the ability to make an effective judgment in the face of numerous factors and complicated conditions.

Rule-based reasoning is to conduct intelligent reasoning for the input fault information by means of human-computer interaction and finally draw a conclusion to assist with the decision-making of fault diagnosis. Rule-based reasoning involves reasoning machine and rule-based knowledge base[6]. Rule-based knowledge base is the set of fault reasoning rules. As the abstraction and model of expert experience, rules are input via front-end page[7]. As the core function of diagnosis, reasoning machine is aimed at interpreting rules, and drawing a reasoning conclusion by means of step-by-step human-computer interaction. The advantage of rule-based reasoning lies in its ability to diagnose the complicated faults in relation to varieties of factors. Below Figure 3 is the flow chart:

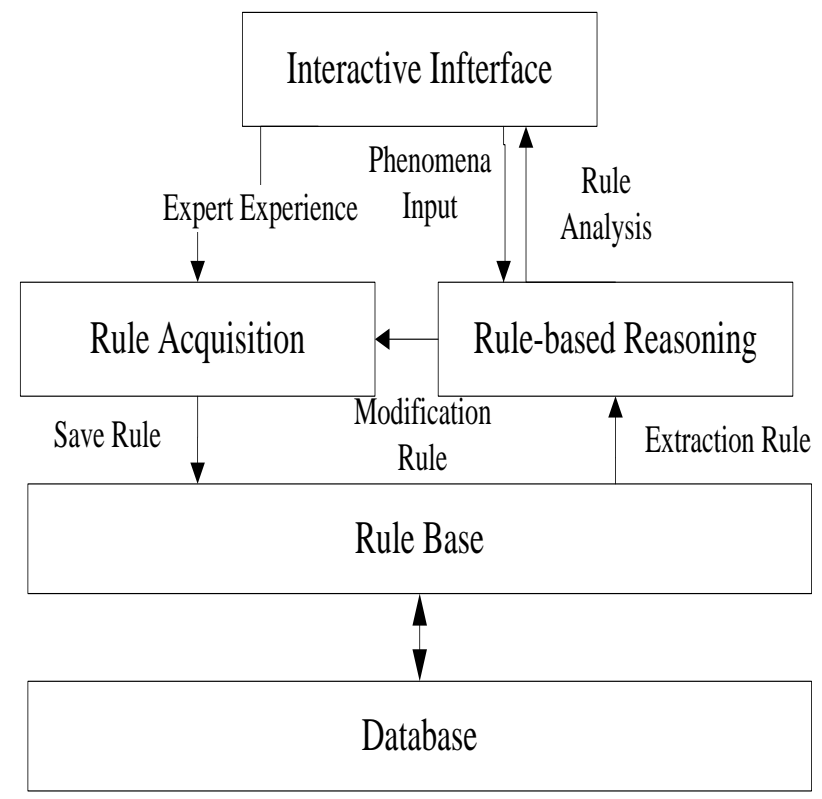

Fig.3. Rule-based Reasoning Process

Statistical analysis is to carry out statistical classification for the existing fault information and display the information with the statistical diagrams such as histogram, pie chart and tendency chart[9]. With the help of statistical analysis, decision makers will understand the category and distribution of fault causes in an intuitive way and then map out corresponding improvement strategies.

When case diagnosis and rule-based reasoning are unable to localize the faults, the remote support function will be triggered, and meanwhile, the remote permission configuration page will pop up from the module. Under this circumstance, there will be a need to define the information 
such as temporary login account number, password, contact information, email, authorization module, and effective login time, and after confirmation, the module will send the login account number, password and effective time via message or email so as to ensure system security.

\section{Conclusion}

In this paper, an event-driven equipment fault operation and maintenance system based on internet remote access is built. This system has implemented the functions as follows: the management over equipment's key parameter data, the reminding and recording of equipment maintenance, and the diagnosis information management over equipment faults. Additionally, this system is equipped with integration interface and therefore can be integrated with corporate information management system as a sub-system.

\section{Acknowledgement}

Foundation item: National High-tech R\&D Program of China (863 Program) “ Combine Harvester Collaborative Key Technology Research and Demonstration Based on the Internet of Things” (2013AA040403-1)

\section{References}

[1] Jaw Link C, “a platform for advanced condition-based health management”. Proceedings of IEEE on Aerospace Conference, 2001, pp. 2909-2914.

[2] Cheng Fan-tien,Yang Haw-ching,Tsai Chia-ying, "Development of a service management scheme for semicon- ductor factory management systems"[c]. Proceedings IEEE International Conference Robotics Automation, 2002, pp.3607-3612.

[3] CUI Wei."'Research and development on fault inform ation processing system for power grids”[D]. Wuhan:Huazhong University of Science and Technology,2004.

[4] Jin-Hua Liu,Guang Tian,Xin-Jie Shao,Jing-Tao Zhou. "Notice of Retraction Application of the fault tree analysis method in the fault diagnosis of breechblock". Quality, Reliability, Risk, Maintenance, and Safety Engineering (QR2MSE), 2013 International Conference Publication Year: 2013, pp.1867-1870.

[5] Qingfeng Wen,Xueping Gu,Wenyun Li. "Information fusion method of multi-data resources and its application to fault diagnosis in power system”. Renewable Power Generation Conference (RPG 2013), 2nd IET Publication Year: 2013 , pp.1-4 On the inference rules.

[6] V. Rybakov, M. Terziler \& C. "Gencer. Unification and Passive Inference Rules for Modal Logics”. Journal of Applied Non-Classical LogicsVolume 10, Issue 3-4, January 2000, pp.369-377. Published online: 30 May 2012.

[7] Nalepa, G.J. (AGH Univ. of Sci. \& Technol., Krakow, Poland), Bobek, S.,Ligeza, A., Kaczor,K.,”Algorithms for Rule Inference in Modularized Rule Bases”. Rule-Based Reasoning, Programming, and Applications. Proceedings 5th International Symposium, RuleML 2011-Europe, pp. 305-312,2011. 